Journal for ImmunoTherapy of Cancer

\section{Impact of a preceding radiotherapy on the outcome of immune checkpoint inhibition in metastatic melanoma: a multicenter retrospective cohort study of the DeCOG}

To cite: Knispel S, Stang A, Zimmer L, et al. Impact of a preceding radiotherapy on the outcome of immune checkpoint inhibition in metastatic melanoma: a multicenter retrospective cohort study of the DeCOG. Journal for ImmunoTherapy of Cancer 2020;8:e000395. doi:10.1136/ jitc-2019-000395

- Additional material is published online only. To view please visit the journal online (http://dx.doi.org/10.1136/jitc2019-000395).

Accepted 30 March 2020

Check for updates

(C) Author(s) (or their employer(s)) 2020. Re-use permitted under CC BY-NC. No commercial re-use. See rights and permissions. Published by BMJ.

For numbered affiliations see end of article.

Correspondence to Professor Selma Ugurel; selma.ugurel@uk-essen.de

\section{ABSTRACT}

Background Immune checkpoint inhibition (ICI) is an essential treatment option in melanoma. Its outcome may be improved by a preceding radiation of metastases. This study aimed to investigate the impact of a preceding radiotherapy on the clinical outcome of $\mathrm{ICI}$ treatment. Methods This multicenter retrospective cohort study included patients who received anti-cytotoxic T-lymphocyte-associated protein 4 (CTLA-4) or antiprogrammed cell death protein 1 (PD-1) ICl with or without preceding radiotherapy for unresectable metastatic melanoma. ICI therapy outcome was measured as best overall response (BOR), progression-free (PFS) and overall survival (OS). Response and survival analyses were adjusted for confounders identified by directed acyclic graphs. Adjusted survival curves were calculated using inverse probability treatment weighting.

Results 835 patients who received ICI (anti-CTLA-4, $\mathrm{n}=596$; anti-PD-1, $\mathrm{n}=239$ ) at 16 centers were analyzed, whereof 235 received a preceding radiotherapy of metastatic lesions in stage IV disease. The most frequent organ sites irradiated prior to ICI therapy were brain (51.1\%), lymph nodes (17.9\%) and bone (17.9\%). After multivariable adjustment for confounders, no relevant differences in $\mathrm{ICl}$ therapy outcome were observed between cohorts with and without preceding radiotherapy. BOR was $8.7 \%$ vs $13.0 \%$ for anti-CTLA-4 (adjusted relative risk $(\mathrm{RR})=1.47 ; 95 \% \mathrm{Cl}=0.81$ to $2.65 ; \mathrm{p}=0.20)$, and $16.5 \%$ vs $25.3 \%$ for anti-PD-1 ( $\mathrm{RR}=0.93 ; 95 \% \mathrm{Cl}=0.49$ to 1.77 ; $\mathrm{p}=0.82$ ). Survival probabilities were similar for cohorts with and without preceding radiotherapy, for anti-CTLA-4 (PFS, adjusted $\mathrm{HR}=1.02,95 \% \mathrm{Cl}=0.86$ to $1.25, \mathrm{p}=0.74$; OS, $\mathrm{HR}=1.08,95 \% \mathrm{Cl}=0.81$ to $1.44, \mathrm{p}=0.61$ ) and for antiPD-1 (PFS, HR=0.84, 95\% $\mathrm{Cl}=0.57$ to $1.26, p=0.41 ; 0 S$, $\mathrm{HR}=0.73,95 \% \mathrm{Cl}=0.43$ to $1.25, \mathrm{p}=0.26)$. Patients who received radiation last before $\mathrm{ICl}(\mathrm{n}=137)$ revealed no better survival than those who had one or more treatment lines between radiation and start of $\mathrm{ICI}(\mathrm{n}=86)$. In 223 patients with brain metastases, we found no relevant survival differences on $\mathrm{ICl}$ with and without preceding radiotherapy.

Conclusions This study detected no evidence for a relevant favorable impact of a preceding radiotherapy on anti-CTLA-4 or anti-PD-1 ICI treatment outcome in metastatic melanoma.

\section{BACKGROUND}

The systemic therapy of metastatic melanoma recently underwent considerable changes due to the introduction of antibodies blocking the immune checkpoints CTLA-4 and PD-1. ${ }^{2}$ Both CTLA-4 (ipilimumab) and PD-1 (nivolumab, pembrolizumab) immune checkpoint inhibitors (ICIs) have been shown to induce durable tumor responses and long-term survival in a subset of patients. ${ }^{3-5}$ Immunological conditions reflecting an inflamed state of a patient's tumor such as expression of PD-L1 or presence of tumor-infiltrating lymphocytes were identified as predictive markers of ICI treatment response. ${ }^{6}$ Due to the high proportion of patients with melanoma presenting a primary resistance to ICI therapy, various strategies have been considered to enhance the tumor's upfront ICI sensitivity. Herein, one promising approach that has been discussed vigorously is the enhancement of tumor immunogenicity by radiation. For various cancer entities including non-smallcell lung cancer (NSCLC) ${ }^{8}{ }^{9}$ head-and-neck cancer $^{10}{ }^{\text {colon }}$ carcinoma $^{11}$ and sarcoma, ${ }^{12}$ it has been demonstrated that radiotherapy 
of tumor lesions generates immune-related abscopal antitumor effects. These effects have been assumed to be mediated by the modulation of the tumor and its microenvironment toward an increased antigen presentation and recognition, leading to an overall improvement of antitumorous immune responses. ${ }^{13}{ }^{14}$ In this regard, radiation and ICI therapy were shown to act synergistically in mouse models as well as in clinical trials. ${ }^{915-17}$ A subgroup analysis of the KEYNOTE-001 trial cohort investigating pembrolizumab in metastatic NSCLC showed prolonged survival times in patients who received a radiotherapy of tumor lesions at any time preceding the start of pembrolizumab. ${ }^{8}$ In melanoma, a variety of small studies and case series led to contradictory results with regard to an effect of radiotherapy on ICI treatment outcome (for review see Ref. 18 and tables 1 and 2). Statistical analyses of larger patient populations that address confounding are lacking, and randomized controlled trials (RCTs) on this topic are difficult to implement.

The present study aimed at investigating the impact of a preceding therapeutic radiation of metastatic lesions on ICI treatment outcome in terms of response and survival on CTLA-4 or PD-1 inhibition in a large multicenter cohort of patients with metastatic melanoma. Since radiotherapy for melanoma is mainly applied to prognostically poor patient groups with advanced metastatic disease such as metastasis to the brain or bone, this study carefully addressed confounding by the use of directed acyclic graphs (DAGs) and inverse probability treatment weighting (IPTW).

\section{METHODS}

We performed a PubMed search for articles published from January 2011 until April 2019. Our search focused on clinical studies investigating a combination or sequencing of radiotherapy and ICI in patients with metastatic melanoma. We selected studies which investigated at least 20 patients, and which reported tumor response, progression-free survival (PFS) or overall survival (OS) as a study endpoint. The used search terms were "melanoma" AND "radiotherapy", "radiation", "radiosurgery" AND "PD-1", "CTLA-4", "ipilimumab", "nivolumab", and "pembrolizumab". The hereby found reported evidence on radiotherapy combined or sequenced with ICI in melanoma was based on a variety of small clinical studies with low patient numbers (tables 1 and 2). The study results were contradictory, and multivariable adjustments to confounders were lacking in the majority of studies. Data from retrospective studies led to heterogeneous results with some studies finding a beneficial effect of radiotherapy on the outcome of ICI and others do not. Data from RCTs comparing patients treated with immune checkpoint inhibitors with and without radiotherapy were missing. Thus, the clinical impact of a preceding radiotherapy on the treatment outcome of ICI in metastatic melanoma was unclear.

\section{Study design}

This multicenter retrospective cohort study was designed to analyze the impact of a preceding radiotherapy on the treatment outcome of ICI with either anti-CTLA-4 or anti-PD-1 monotherapy in patients with advanced metastatic melanoma. The secondary objective was to analyze this impact in a subgroup of patients with brain metastases. Patient selection criteria were histologically proven diagnosis of melanoma, treatment with single agent anti-CTLA-4 or anti-PD-1 ICI for unresectable metastatic disease of stage IV according to American Joint Committee on Cancer (AJCC)-v8 criteria, ${ }^{19}$ ICI therapy start between January 2010 and June 2017, complete documentation of all treatment lines applied before ICI, and at least one documented follow-up visit after start of ICI therapy. The participating study centers were skin cancer centers of the German Dermatologic Cooperative Oncology Group (DeCOG).

\section{Patient registry}

Patients were identified at the participating centers according to the abovementioned selection criteria. Data were extracted from patient files, collected on standardized electronic case record forms, and merged in one central electronic registry. The following known prognostic factors of stage IV melanoma were collected: sites of metastasis categorized by AJCC-v8 $8{ }^{19}$ patients' overall performance status (OPS) graded by Eastern Cooperative Oncology Group categories, and serum lactate dehydrogenase activity at baseline of ICI therapy. All treatment lines received in stage IV disease prior to the investigated ICI therapy were assessed and categorized by type of treatment (radiotherapy, chemotherapy, kinase inhibition, immunotherapy). Details on radiotherapy were recorded including the type of radiation and the irradiated organ site. Outcome parameters of ICI treatment were collected, including best overall response (BOR), PFS, and OS survival. BOR was defined as best response recorded from the start of ICI until disease progression and was evaluated according to response evaluation criteria in solid tumors (RECIST)v1.1. ${ }^{20} \mathrm{PFS}$ and OS were measured from ICI therapy start until disease progression or death, respectively; if no such event occurred, the date of last patient contact was used as endpoint of survival assessment (censored observation).

\section{Data analysis}

Survival curves and median survival times with 95\% CIs were calculated using the Kaplan-Meier method for censored failure time data. The multivariable Cox proportional hazards model was applied to estimate the raw and the multivariably adjusted effects of a preceding radiotherapy on PFS and OS of patients treated with ICI in terms of HRs. Adjustment for multiple confounders was done by IPTW. ${ }^{21}$ To illustrate the effect of IPTW on the baseline characteristics of patient groups being compared, see online supplementary tables 1 and 2. The proportional hazards assumption was tested using the 


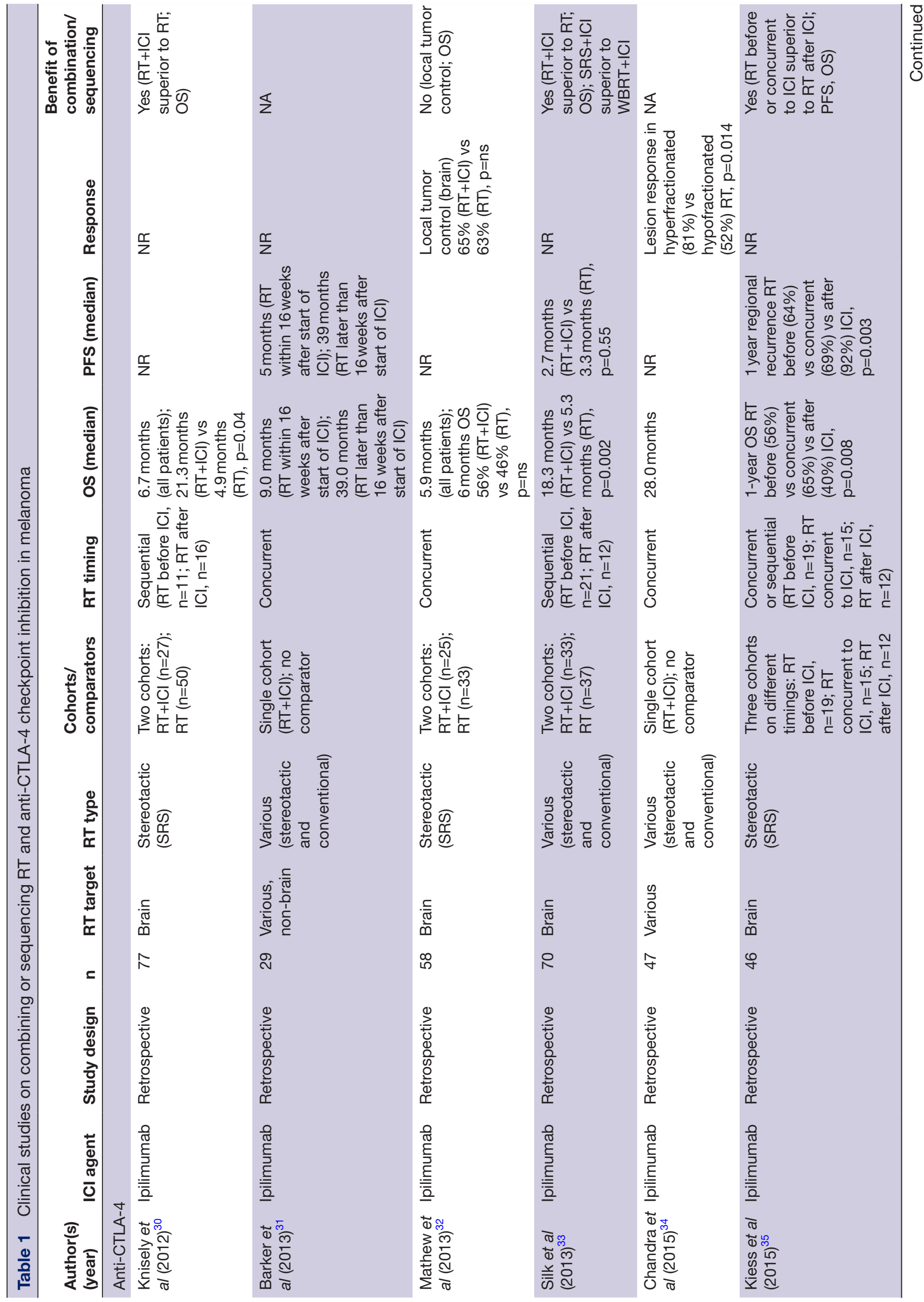




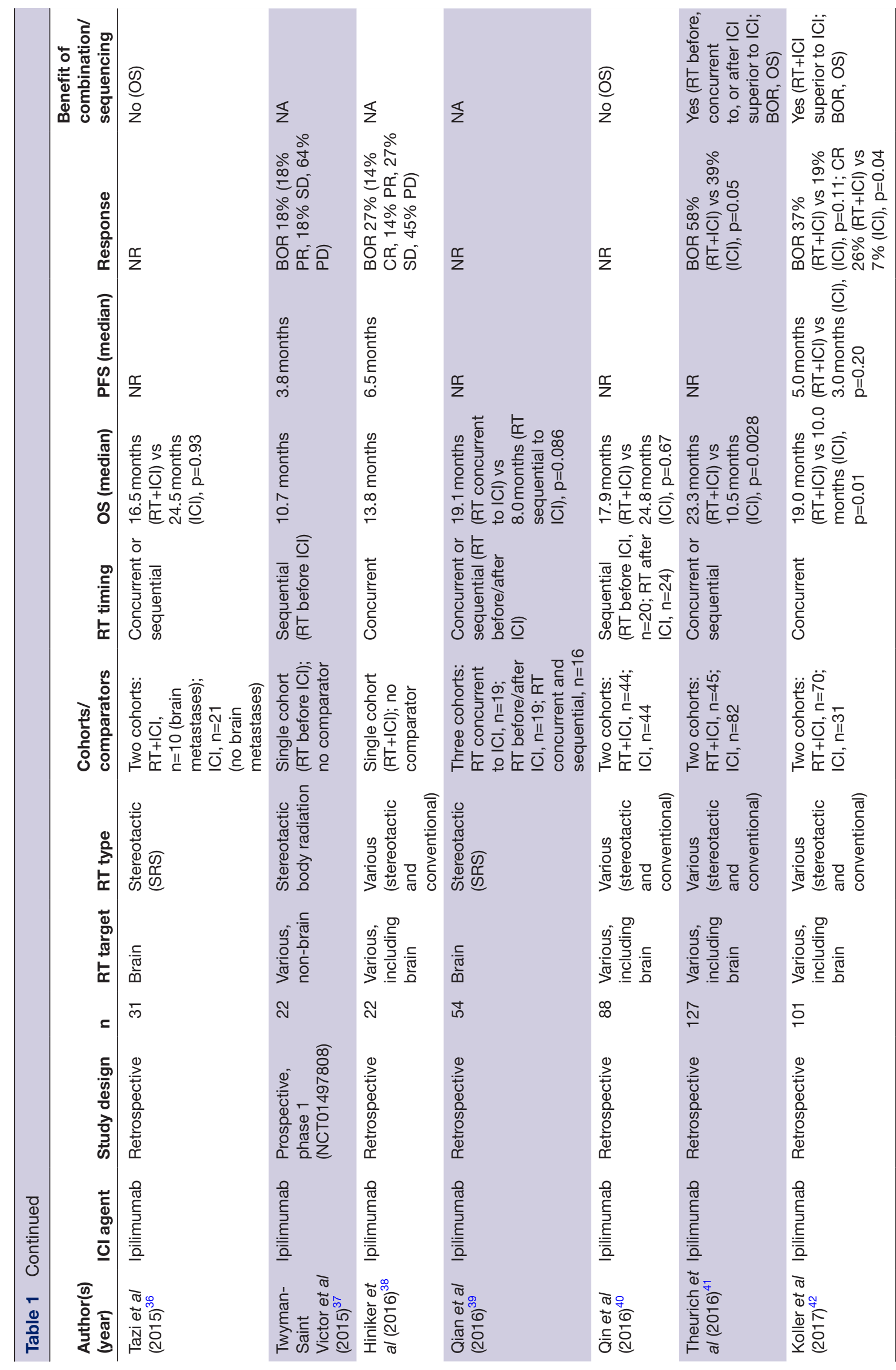




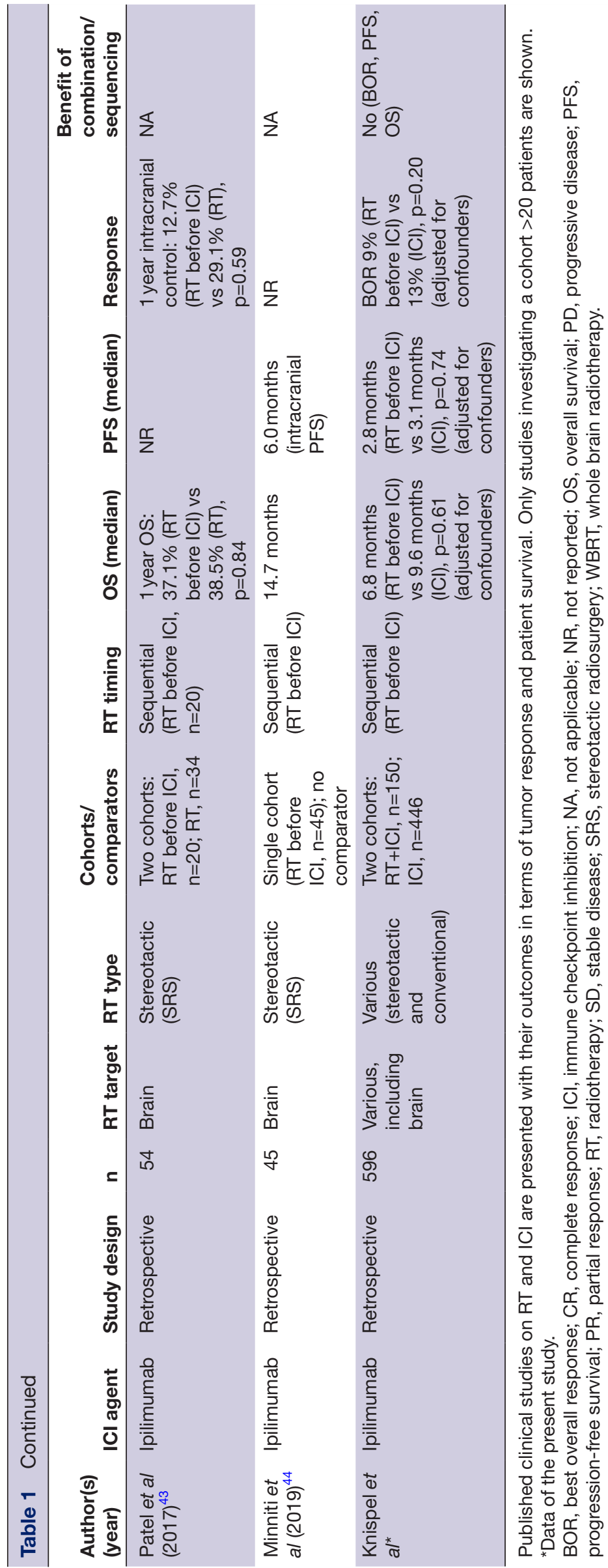




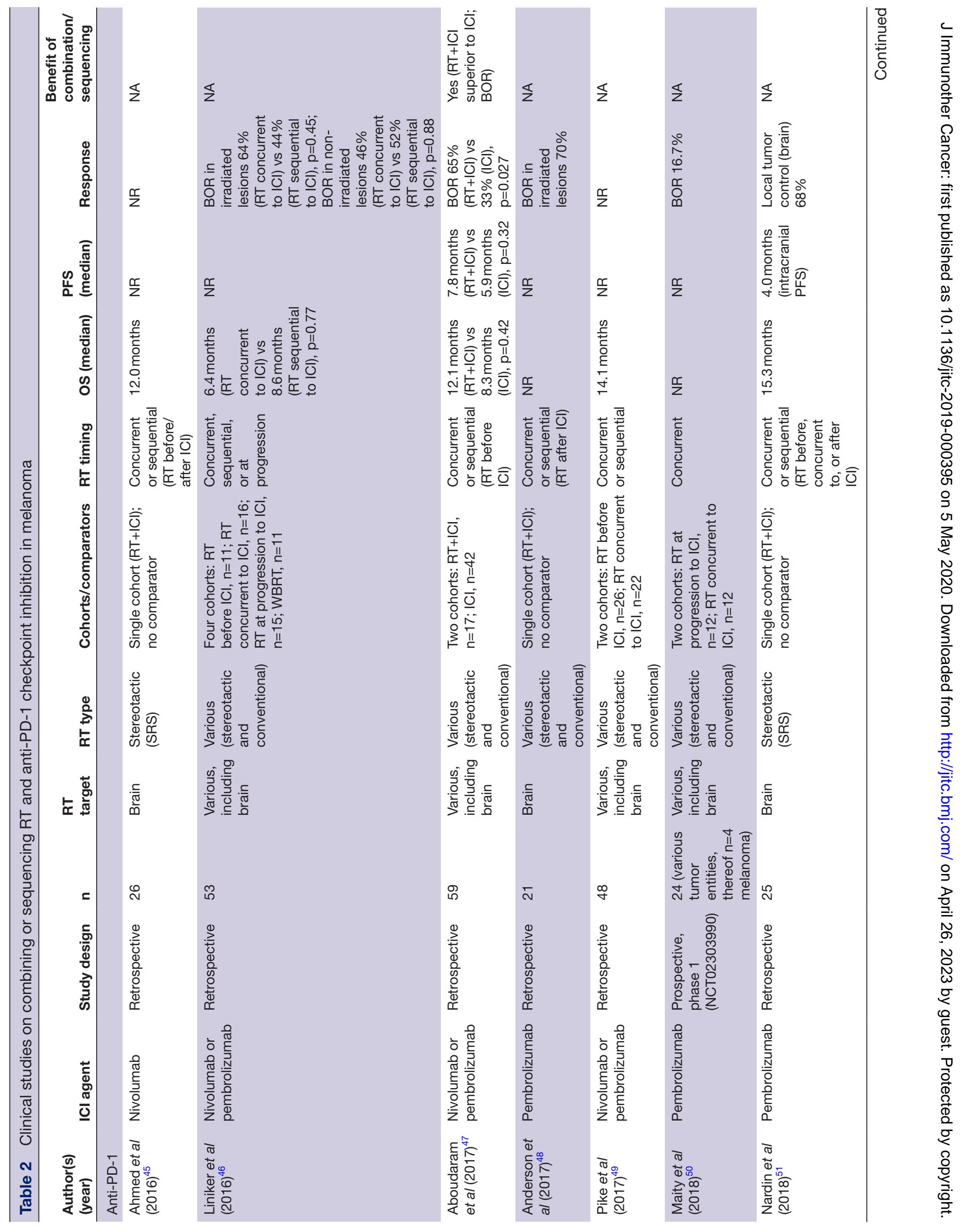




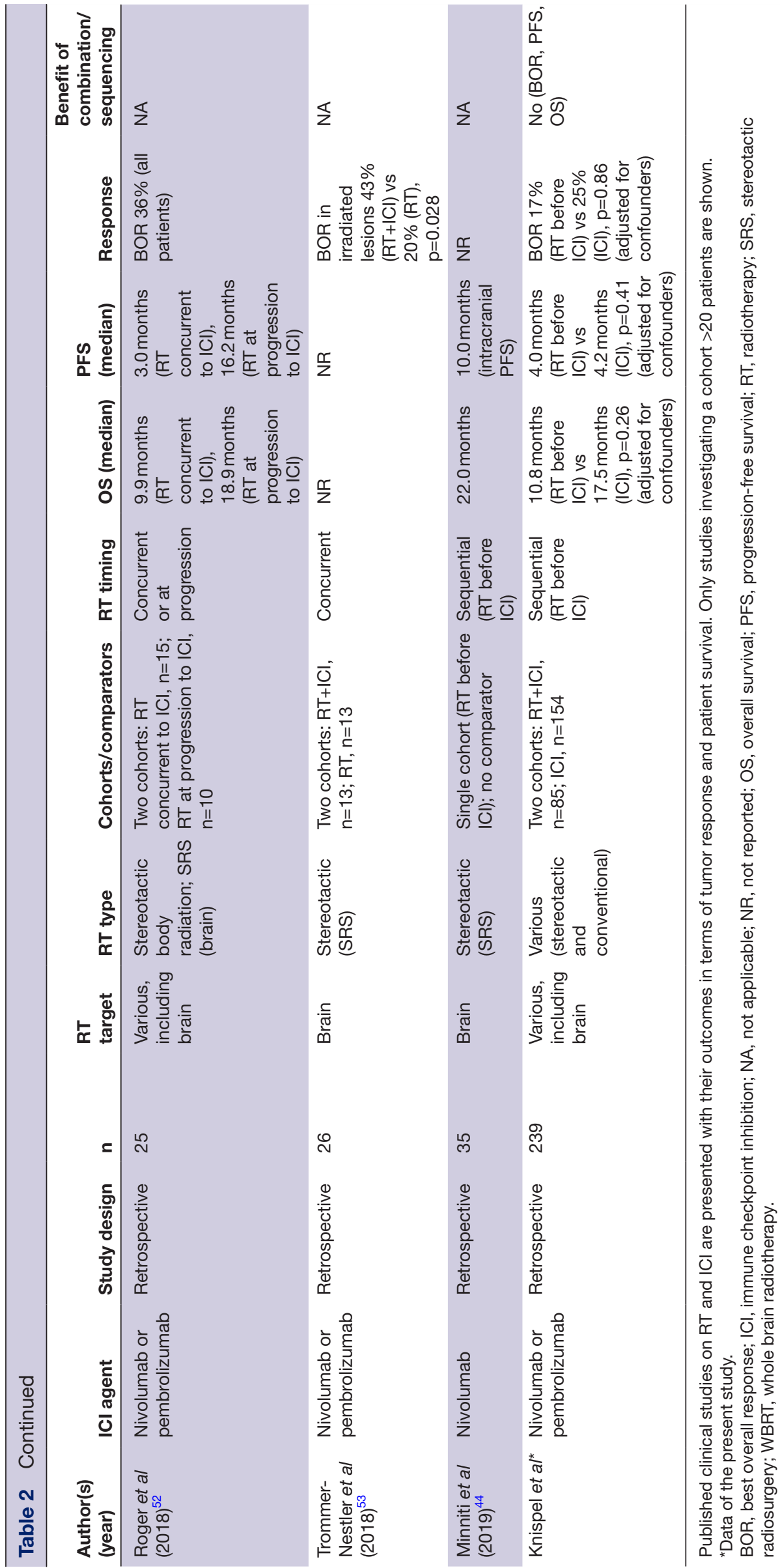



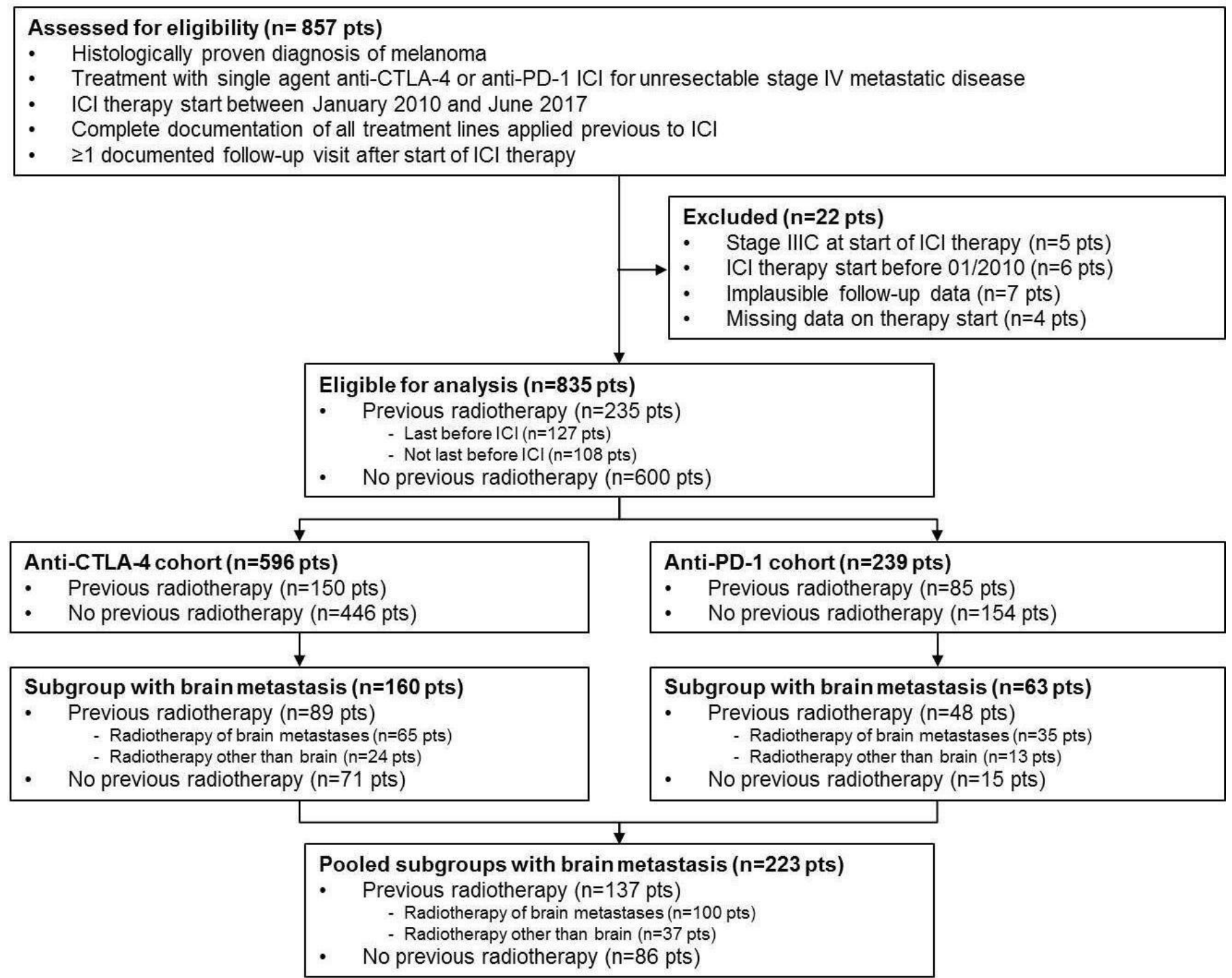

Figure 1 Schematic presentation of the patient selection flow. Eight hundred and fifty-seven patients were identified at 16 clinical centers. Thereof, 835 patients were eligible for analysis, including a subgroup of 223 patients with brain metastasis. ICl, immune checkpoint inhibition.

supremum test. Log-binomial regression models were used to estimate crude and multivariably adjusted relative risks (RR) and 95\% CI for BOR. To define the confounder adjustment sets, we used DAGs. ${ }^{22}$ The adjusted survival curves were estimated using IPTW in the Cox models. We calculated and reported CIs to assess the precision of our estimates. All presented p-values are two-tailed and unadjusted for potential multiple comparisons to allow a hypothesis-generating exploratory data analysis.

\section{RESULTS}

\section{Patient characteristics}

Data of 857 consecutive patients from 16 centers were entered into the central registry. Thereof, 22 patients had to be excluded from analysis (figure 1). The remaining patient population $(\mathrm{n}=835)$ was used for all further analysis. Detailed patient characteristics at ICI therapy start are presented in table 3. Data on the patients' systemic pretreatment in stage IV are given in online supplementary table 3 .

\section{Radiotherapy}

Of all patients eligible for analysis, 235 (28.2\%) received radiotherapy of at least one metastatic lesion in stage IV at any time preceding the start of the investigated ICI. Radiotherapy applied in an adjuvant setting to lymph node basins in stage III disease was not considered. Patients who received radiotherapy concurrent to ICI treatment were not eligible. The most frequent organ sites irradiated prior to ICI therapy were brain $(51.1 \%)$, lymph nodes $(17.9 \%)$, and bone (17.9\%). Among patients with a preceding radiotherapy of the brain $(n=100)$, the most common radiation type was whole brain radiation $(44.0 \%)$, followed by stereotactic radiation $(33.0 \%)$. With regard to ICI therapy type, $150(25.2 \%)$ of 596 patients who received anti-CTLA-4, and 85 (35.6\%) of 239 patients treated with anti-PD-1, respectively, received a preceding 
Table 3 Patient characteristics at start of ICI

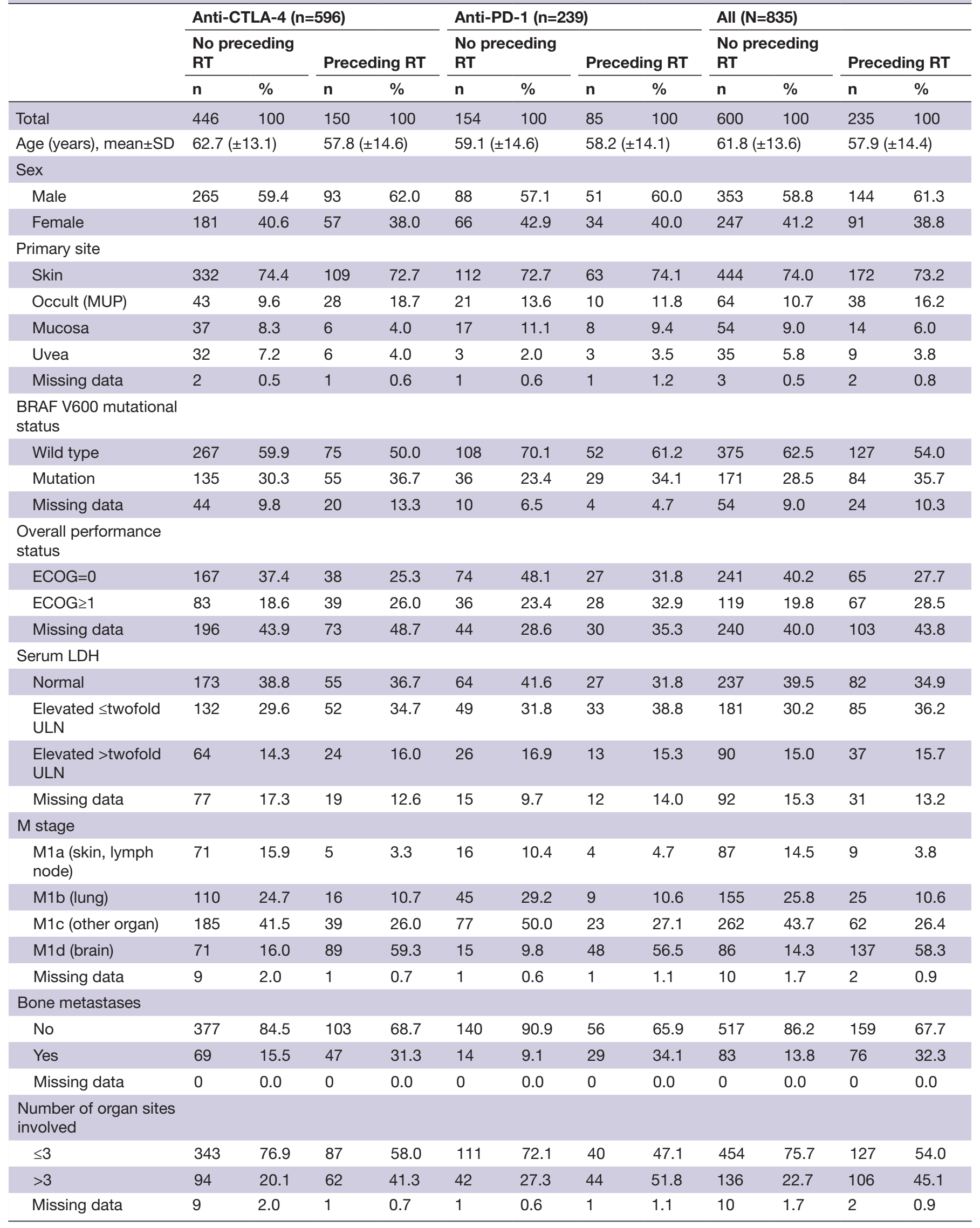


Table 3 Continued

\begin{tabular}{|c|c|c|c|c|c|c|c|c|c|c|c|c|}
\hline & \multicolumn{4}{|c|}{ Anti-CTLA-4 $(n=596)$} & \multicolumn{4}{|c|}{ Anti-PD-1 ( $n=239)$} & \multicolumn{4}{|c|}{ All $(\mathrm{N}=835)$} \\
\hline & \multicolumn{2}{|c|}{$\begin{array}{l}\text { No preceding } \\
\text { RT }\end{array}$} & \multicolumn{2}{|c|}{ Preceding RT } & \multicolumn{2}{|c|}{$\begin{array}{l}\text { No preceding } \\
\text { RT }\end{array}$} & \multicolumn{2}{|c|}{ Preceding RT } & \multicolumn{2}{|c|}{$\begin{array}{l}\text { No preceding } \\
\text { RT }\end{array}$} & \multicolumn{2}{|c|}{ Preceding RT } \\
\hline & $\mathbf{n}$ & $\%$ & $\mathbf{n}$ & $\%$ & $\mathbf{n}$ & $\%$ & $\mathbf{n}$ & $\%$ & $\mathbf{n}$ & $\%$ & $\mathbf{n}$ & $\%$ \\
\hline \multicolumn{13}{|l|}{$\begin{array}{l}\text { Systemic pretreatment } \\
\text { in stage IV }\end{array}$} \\
\hline No & 138 & 30.9 & 27 & 18.0 & 28 & 18.2 & 11 & 13.0 & 166 & 27.7 & 38 & 16.2 \\
\hline Missing data & 17 & 3.9 & 0 & 0.0 & 3 & 2.0 & 2 & 2.3 & 20 & 3.3 & 2 & 0.8 \\
\hline $\begin{array}{l}\text { Duration of stage IV } \\
\text { disease at start of ICI } \\
\text { (months) } \\
\text { Median (p10; p90) }\end{array}$ & \multicolumn{2}{|c|}{$\begin{array}{l}6.0 \\
(0.8 ; 26.6)\end{array}$} & \multicolumn{2}{|c|}{$\begin{array}{l}9.8 \\
(2.2 ; 42.4)\end{array}$} & \multicolumn{2}{|c|}{$\begin{array}{l}13.7 \\
(1.4 ; 36.2)\end{array}$} & \multicolumn{2}{|c|}{$\begin{array}{l}14.2 \\
(2.5 ; 43.8)\end{array}$} & \multicolumn{2}{|c|}{$\begin{array}{l}8.2 \\
(1.4 ; 26.5)\end{array}$} & \multicolumn{2}{|c|}{$\begin{array}{l}14.7 \\
(4.5 ; 68.2)\end{array}$} \\
\hline
\end{tabular}

The given patient characteristics refer to the start of the investigated ICI therapy. Percentages are given per column for each individual patient cohort. M stage categories refer to the AJCC v8 classification system; the number of organ sites refer to organs involved with metastasis; systemic pretreatment describes systemic therapies received by the patient for inoperable stage IV disease (not adjuvant) prior to the investigated ICl therapy.

BRAF, v-raf murine sarcoma viral oncogene homolog B1; ECOG, Eastern Cooperative Oncology Group; ICI, immune checkpoint inhibition; LDH, lactate dehydrogenase; MUP, melanoma of unknown primary; p10, 10th percentile; p90, 90th percentile.; RT, radiotherapy; ULN, upper limit of normal.

radiotherapy (online supplementary table 4). The patient cohorts with and without preceding radiotherapy differed markedly in important prognostic factors such as OPS, sites of metastasis (particularly brain), number of metastatic sites, and systemic pretreatment in stage IV, revealing a less favorable prognosis for patients who received radiotherapy before start of ICI in both the antiCTLA-4 and the anti-PD-1 therapy groups.

\section{Statistical adjustment sets}

After application of DAGs, separate adjustment sets of confounders were defined for PFS and OS, each for the total patient population as well as for the subgroup of patients with brain metastases (online supplementary figure 1). The adjustment set for the response parameter BOR equals than that for PFS. For the total patient population, the adjustment set included brain metastases (yes vs no), bone metastases (yes vs no), primary site of disease (categorical), and systemic pretreatment (yes vs no) for PFS and BOR, and duration in stage IV (continuous), brain metastases, bone metastases, primary site of disease, BRAF mutational status (yes vs no), and systemic pretreatment for OS. For patients with brain metastases, the confounders were primary site of disease and systemic pretreatment for PFS and BOR, and duration in stage IV, primary site of disease, BRAF mutational status, and systemic pretreatment for OS.

\section{Survival outcome of ICI therapy}

The closing date for the patient registry was February 15, 2018. At that time, the total study population had a median follow-up time of 13.1 months. $78.7 \%$ of the patients had experienced disease progression and $55.7 \%$ had died.
For patients treated with anti-CTLA-4, the median PFS was 2.9 months ( $95 \% \mathrm{CI}=2.0$ to 5.4 months), and the median OS was 6.2 months ( $95 \% \mathrm{CI}=2.8$ to 12.0 months). After multivariable adjustment for confounders, no difference could be detected for PFS (HR=1.02; 95\% CI $=0.86$ to 1.25; $\mathrm{p}=0.74$; figure $2 \mathrm{~A})$ and $\mathrm{OS}(\mathrm{HR}=1.08 ; 95 \% \mathrm{CI}=0.81$ to $1.44 ; \mathrm{p}=0.61$; figure $2 \mathrm{D}$ ) between patients who received a preceding radiotherapy before CTLA-4 inhibition and patients who did not.

For patients treated with anti-PD-1, the median PFS was 3.8 months ( $95 \% \mathrm{CI}=1.9$ to 6.2 months), and the median OS was 13.0 months (95\% CI=3.7 to 18.0 months). A radiotherapy preceding anti-PD-1 treatment had no impact on PFS (HR $=0.84 ; 95 \% \mathrm{CI}=0.57$ to $1.26 ; \mathrm{p}=0.41$; figure $2 \mathrm{~B}$ ) or OS $(\mathrm{HR}=0.73 ; 95 \% \mathrm{CI}=0.43$ to $1.25 ; \mathrm{p}=0.26$; figure $2 \mathrm{E}$ ) after adjustment for confounders. Subgroup analysis of patients with $(n=192)$ or without $(n=45)$ pretreatment with anti-CTLA-4 prior to anti-PD-1 ICI revealed a possible survival benefit of a previous radiotherapy in patients treated with anti-PD-1 without prior treatment with antiCTLA-4 after adjustment for confounders (online supplementary figure 2B,D). This benefit was more visible in OS (HR 0.36; 95\% CI 0.08 to $1.57 ; \mathrm{p}=0.17$ ) than in PFS (HR $0.91 ; 95 \%$ CI 0.47 to $1.75 ; \mathrm{p}=0.78$ ); however, due to the small sample size, the effect estimation did not provide meaningful results. In patients treated with anti-CTLA-4 prior to anti-PD-1 ICI, no differences were visible for PFS and $\mathrm{OS}$ between patients with or without preceding radiotherapy (online supplementary figure 2A,C).

To analyze a potential effect of different timings of radiation in relation to the investigated ICI, the total number of patients who received radiotherapy prior to anti-CTLA-4 or anti-PD-1 ( $\mathrm{n}=235)$ were divided into those 

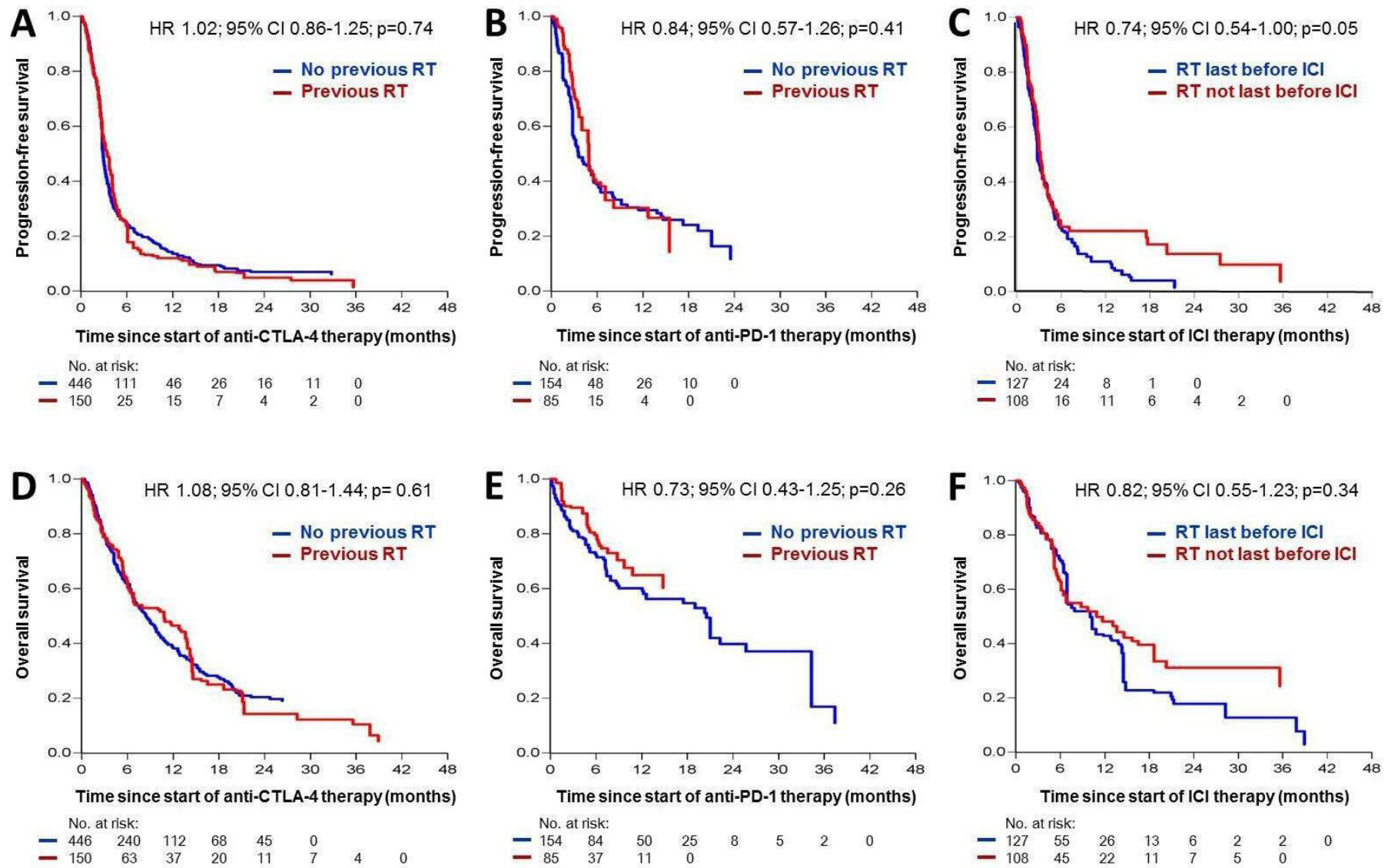

Figure 2 Kaplan-Meier curves showing the probability of progression-free (A-C) and overall survival (D-F) adjusted for confounding factors in patients with metastatic melanoma treated with immune checkpoint inhibitors (ICls). $A$ and $D: n=596$ patients treated with anti-CTLA-4 ICI with or without preceding radiotherapy; $B$ and $E: n=239$ patients treated with anti-PD-1 $\mathrm{ICl}$ with or without preceding radiotherapy; $\mathrm{C}$ and $\mathrm{F}: \mathrm{n}=235$ patients treated with anti-CTLA-4 or anti-PD-1 ICI and preceding radiotherapy, either last before $\mathrm{ICI}$ or with at least one treatment line between radiotherapy and start of ICI. RT, radiotherapy.

who had radiotherapy last before start of ICI $(\mathrm{n}=127$; $54 \%)$, and those who had one or more treatment lines between radiation and ICI $(n=108 ; 46 \%)$. Within the first 6 months after ICI start, no difference was observed for multivariably adjusted PFS and OS between both groups. However, at 6 months or later, those patients who received radiotherapy last before ICI showed a poorer survival outcome (PFS, $\mathrm{HR}=0.74,95 \% \mathrm{CI}=0.54$ to 1.00 , $\mathrm{p}=0.05 ; \mathrm{OS}, \mathrm{HR}=0.82,95 \% \mathrm{CI}=0.55$ to $1.23, \mathrm{p}=0.34$ ), figure $2 \mathrm{C}, \mathrm{F}$ ).

\section{Response outcome of ICI therapy}

After multivariable adjustment for confounders, no relevant differences were observed for objective response $(\mathrm{CR}+\mathrm{PR})$ between patients who underwent a preceding radiotherapy and patients who did not, both in the antiCTLA-4 $(8.7 \%$ vs $13.0 \%$; RR $=1.47 ; 95 \%$ CI $=0.81$ to 2.65 ; $\mathrm{p}=0.20)$ and in the anti-PD-1 ICI therapy cohort $(16.5 \%$ vs $25.3 \%$; $\mathrm{RR}=0.93 ; 95 \% \mathrm{CI}=0.49$ to $1.77 ; \mathrm{p}=0.82$ ); table 4 . Also, the individual categories of BOR showed no differences between groups with or without preceding radiotherapy (table 4).

\section{Patients with brain metastases}

$26.7 \%$ ( $n=223$ ) of the total patient population had brain metastases at start of the investigated ICI, whereof $61 \%$ $(\mathrm{n}=137)$ received a preceding radiotherapy (table 3, figure 1). Due to the clinical relevance of the presence of brain metastases and the high frequency of radiotherapy applied in these patients, this subgroup was investigated separately for a potential impact of a preceding radiotherapy on ICI treatment outcome. After multivariable adjustment for confounders, no relevant differences were observed for PFS (HR= $0.85 ; 95 \% \mathrm{CI}=0.63$ to $1.15 ; \mathrm{p}=0.29$; figure $3 \mathrm{~A})$ or $\mathrm{OS}(\mathrm{HR}=0.77 ; 95 \% \mathrm{CI}=0.53$ to $1.13 ; \mathrm{p}=0.18$; figure $3 \mathrm{C}$ ). With regard to the type of brain radiation, we found no survival differences between whole brain radiation and stereotactic brain radiation (PFS, HR $=0.97,95 \%$ $\mathrm{CI}=0.80$ to $1.17, \mathrm{p}=0.74$; OS, $\mathrm{HR}=1.05,95 \% \mathrm{CI}=0.72$ to 1.52, $\mathrm{p}=0.80$; figure $3 \mathrm{~B}, \mathrm{D})$. Moreover, patients with brain metastases showed no difference in objective response to ICI between groups with and without preceding radiation (8.8\% vs $3.5 \%$; $\mathrm{RR}=2.54 ; 95 \% \mathrm{CI}=0.73$ to $8.75 ; \mathrm{p}=0.14$; table 4$)$. 
Table 4 Therapy outcome of ICI

Total patient population $(\mathrm{N}=835)$

Patients with brain metastases ( $N=223)$

Anti-CTLA-4 ( $n=596)$

Anti-PD-1 ( $n=239)$

Anti-CTLA-4 (n=160)/anti-PD-1

No $(n=63)$

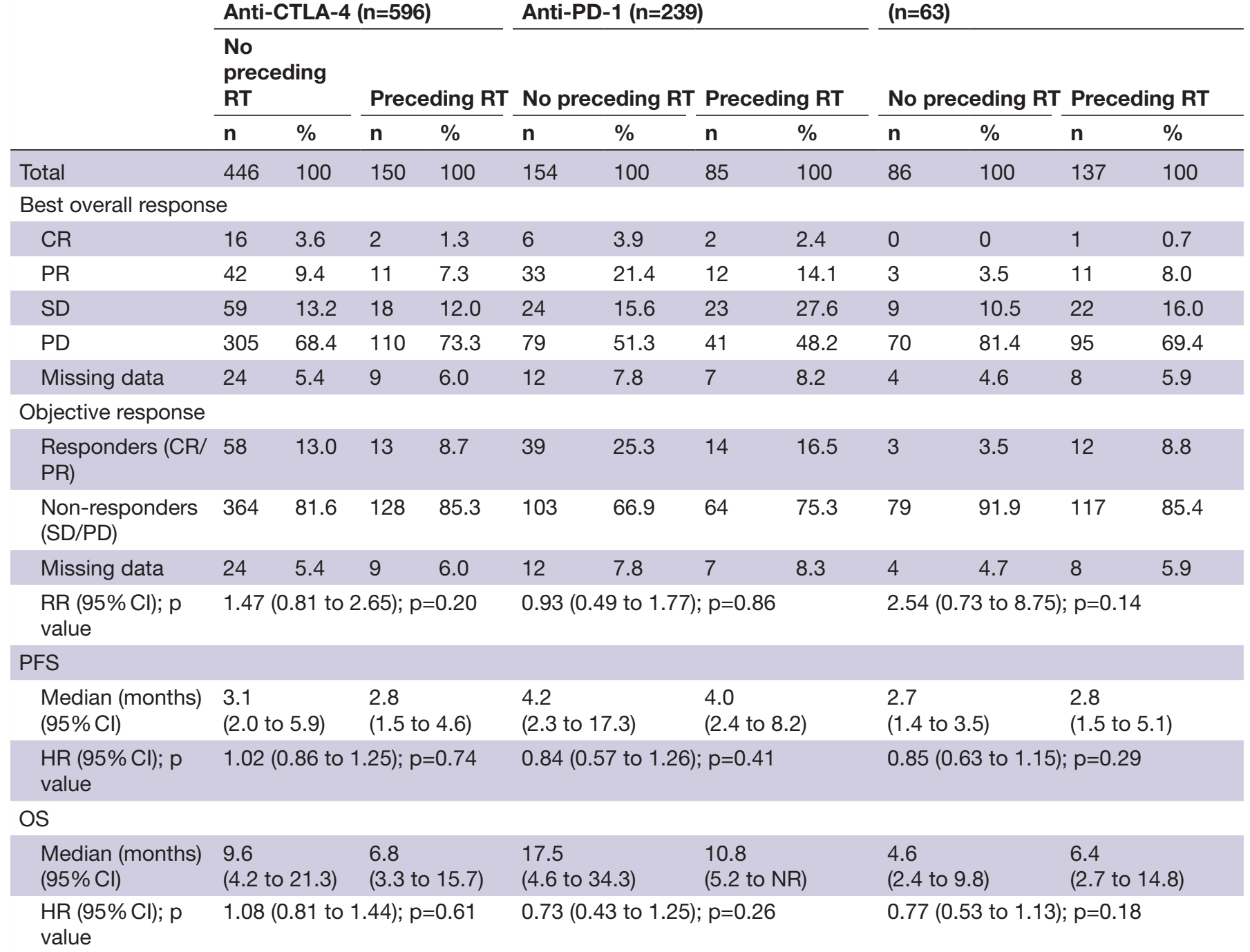

Therapy outcome of $\mathrm{Cl}$ is shown separately for patients treated with anti-CTLA-4 and for patients treated with anti-PD-1, as well as for a subgroup of patients with brain metastases (M1d) treated with either anti-CTLA-4 or anti-PD-1. The effect of a preceding radiotherapy on response to $\mathrm{ICl}$ is given as $\mathrm{RR}$ to achieve an objective response; the effect of a preceding radiotherapy on survival on ICI therapy is given as $\mathrm{HR}$ to attain a progression (PFS) or death (OS). All RR, HR, and $p$ values result from multivariable analyses adjusted for confounders.

$\mathrm{ICI}$, immune checkpoint inhibition; NR, not reached; OS, overall survival; PFS, progression-free survival; RR, relative risk.

\section{DISCUSSION}

Radiation is a common treatment modality in metastatic melanoma, primarily aimed at the control of difficult to treat or symptomatic metastatic sites such as brain or bone. Since there are no detrimental side effects of interaction described, radiotherapy is often combined or sequenced with immunotherapy in these patients. This combination or sequencing approach gained additional attention from the hypothesis of radiation to act as means of converting immunologically "cold" into "hot" tumor microenvironments, herein functioning as a sensitizer for ICI therapy. ${ }^{1623}$ Whether the radiation of tumor lesions prior to the start of ICI can increase ICI treatment efficacy in patients with melanoma currently is a matter of ongoing investigation and intense debate.

For both CTLA-4 and PD-1 inhibition, several case reports exist on patients with melanoma who experienced impressive tumor responses to ICI when combined or sequenced with radiotherapy. ${ }^{24} 25$ The current evidence on ICI and radiotherapy in melanoma provided by clinical studies on cohorts of $>20$ patients differs between the two types of inhibitors, anti-CTLA- 4 and anti-PD-1 (tables 1 and 2). For anti-CTLA-4, out of 15 studies, 7 did not compare cohorts with and without radiation. Of the remaining eight studies, five demonstrated a prolonged OS $(n=5)$ or an increased BOR $(n=3)$ in patients treated with 

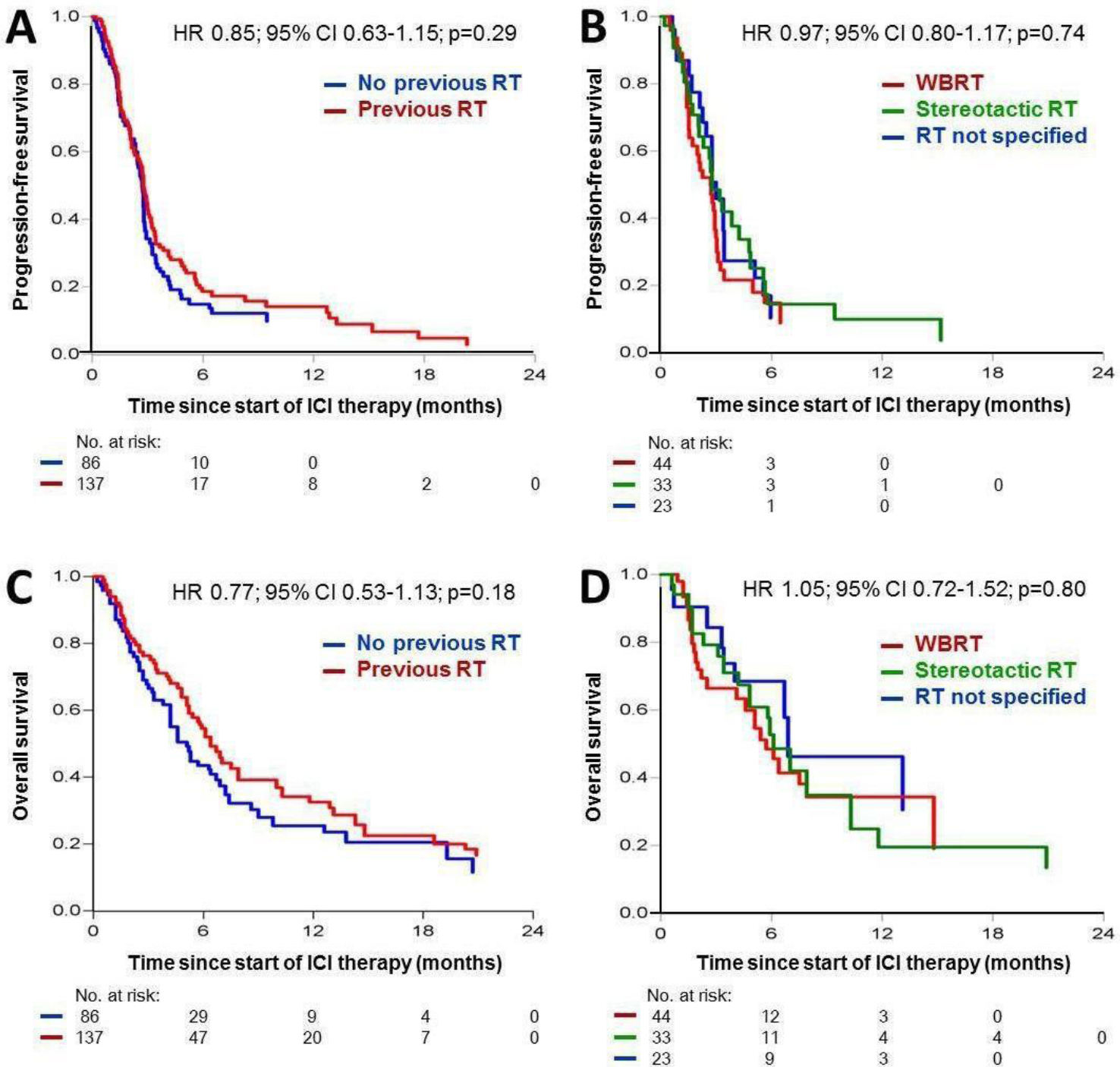

Figure 3 Kaplan-Meier curves showing the probability of progression-free (A, B) and overall survival (C, D) adjusted for confounding factors in patients with melanoma with brain metastases (AJCC stage M1d) treated with anti-CTLA-4 or anti-PD-1 immune checkpoint inhibitors (ICls). A and C: $n=223 \mathrm{M} 1 \mathrm{~d}$ patients treated with $\mathrm{ICl}$ with or without preceding radiotherapy; $\mathrm{B}$ and $\mathrm{D}: \mathrm{n}=100 \mathrm{M} 1 \mathrm{~d}$ patients treated with $\mathrm{ICl}$ and preceding radiotherapy of the brain displayed by the radiation technique used (whole brain radiotherapy (WBRT), stereotactic radiotherapy, or not further specified radiation therapy of brain metastases). RT, radiotherapy.

radiotherapy and ICI combined or sequenced, whereas three studies did not detect such a beneficial effect. For anti-PD-1 ICI combined or sequenced with radiation, out of 10 published studies only 1 compared patient cohorts with and without radiation, revealing a positive impact on BOR but no relevant differences in PFS or OS. The other nine studies investigated different timings and modalities of radiation in relation to anti-PD-1. Notably, approximately half of all studies on radiation and ICI $(8 / 15$ antiCTLA-4; 4/10 anti-PD-1) investigated brain metastases as the only target of radiation, with the majority of these studies focusing on stereotactic radiosurgery. Additionally important, of these 25 clinical studies all but 2 were retrospective. The remaining two prospective studies included small patient numbers only and had no comparator arms. Statistical analyses addressing the important issue of confounders are lacking, mainly due to the small patient numbers analyzed in most of these studies. Taken together, the current clinical data comparing ICI with or without combined or sequenced radiotherapy rely on relatively small retrospective studies, with no implementation of multivariable adjustment for confounders. Results from RCTs adressing this subject are completely missing.

Our present study comprises by far the largest patient cohorts on ICI plus or minus radiation in melanoma to date. In contrast to some of the abovementioned smaller studies, we detected no relevant impact of a preceding radiotherapy on ICI treatment outcome, neither in patients treated with anti-CTLA-4 nor in patients treated with anti-PD-1. In patients treated with anti-PD-1 with no pretreatment with anti-CTLA-4, a possible survival benefit of a previous radiotherapy could not be excluded, while in patients treated with anti-CTLA-4 prior to anti-PD-1, a precedent radiotherapy led to no relevant difference 
in survival. Regarding tumor response in terms of BOR, our results revealed broad CIs indicating a wide range of potential effects of a preceding radiation. In contrast, for survival in terms of PFS and OS, the narrow CIs found by us showed no evidence that radiotherapy relevantly influenced the rate of progression or death.

The observed discrepancy between published data showing a beneficial impact of a preceding radiation on ICI treatment outcome at least in some clinical studies, and our own data revealing no evidence for such an effect can be primarily explained by the small patient numbers investigated within the previously reported studies (see tables 1 and 2). The largest study comprised 127 patients with only 45 thereof receiving radiotherapy; the majority of the remaining studies investigated cohorts of 10 to 30 patients only. These small patient numbers probably led to unreliable results and hampered the application of multivariable adjustment for confounders. Confounder adjustment is of essential importance to obtain valid effect estimates, as it was applied in our present study. Additionally, the multicenter design of our present study reduces center-induced bias, rendering the present study results superior to those emerging from the previously reported oligocentric or monocentric studies. However, the conclusions drawn by us require validation in RCTs. This is particularly true for the further investigation of sequencing and timing of radiotherapy, since the present study included patients with radiotherapy before ICI therapy only, herein excluding patients with radiotherapy concurrent to ICI.

Notably, different from other cancer entities, melanoma has been demonstrated to react to irradiation not only with unrestricted immune activation leading to beneficial abscopal tumor regressions but also with rapidly evolving resistance mechanisms. Thus, Twyman-Saint Victor and coworkers demonstrated that irradiation of melanoma metastases led to an upregulation of PD-L1 expression of tumor cells and to an increased exhaustion of tumorinfiltrating lymphocytes within these lesions, which both are mechanisms enabling the escape of tumor cells from immune recognition. ${ }^{26}$

ICI therapy of melanoma has recently been shown to exert high efficacy also in patients with brain metastases. ${ }^{27} 28$ Our present study cohort comprised a relevant number of patients with brain metastases with or without radiotherapy prior to ICI, allowing us to perform separate analyses of this clinically highly relevant patient population using appropriate adjustment sets of confounders. We did not find a relevant impact of a preceding intracranial radiation on response and survival outcomes of ICI therapy. Moreover, subgroup analyses with regard to the type of preceding intracranial radiotherapy, in terms of conventional whole brain radiation compared with stereotactic radiation, revealed no relevant differences in ICI therapy outcome. However, the wide CIs resulting from our analysis suggest that a possible advantage of a preceding radiotherapy for this patient subgroup cannot be excluded.
Notably, the conclusions drawn from our study results are limited because of its observational nature. The groups of patients with and without previous radiotherapy compared by us differed markedly in their patient and treatment characteristics, which we tried to compensate by multivariate adjustment for confounders. Also, nearly half of the investigated patients who received a preceding radiotherapy had this radiation applied not immediately before start of ICI therapy, but earlier in stage IV disease with at least one intermittent treatment line. However, a comparison of both groups did not show a benefit in ICI treatment outcome for those patients who were irradiated immediately before ICI, but rather revealed that these patients had a poorer ICI therapy outcome. Additionally, it is apparent that the patient cohorts investigated by us are characterized by a shorter PFS and OS compared with survival rates from landmark clinical trials. This can mainly be explained by the inferior treatment outcomes in real-world patient populations compared with the highly selected populations investigated in RCTs. ${ }^{29}$

\section{CONCLUSIONS}

The results of the present study indicate no relevant impact of a preceding radiotherapy of metastatic lesions on the therapy outcome of anti-CTLA-4 or anti-PD-1 ICI in metastatic melanoma. Based on our data, the intended use of radiation as a sensitizer for ICI therapy of melanoma can currently not be recommended for routine clinical use. Instead, patients should be preferentially treated within prospective clinical trials adressing the question of synergistic beneficial effects of radiotherapy and ICI, either simultaneously or sequentially applied. There are various RCTs planned or already activated investigating this effect (for overview see Refs 15-17 and https://clinicaltrials.gov/). However, recruitment of patients into these trials is difficult, leading to a slow trial progress with results pending for a longer term. Until data from RCTs become available, the results of our present multicenter cohort study is the most valid information to guide clinicians toward the sequencing of radiotherapy and ICI in metastatic melanoma.

Author affiliations
${ }^{1}$ Department of Dermatology, University Hospital Essen, University of Duisburg-
Essen, Essen, Germany
${ }^{2}$ Center of Clinical Epidemiology, Institute for Medical Informatics, Biometry and
Epidemiology, University of Duisburg-Essen, Essen, Germany
${ }^{3}$ Skin Cancer Center Hannover, Department of Dermatology, Hannover Medical
School, Hannover, Germany
${ }^{4}$ Department of Dermatology, University Hospital Erlangen, Erlangen, Germany
${ }^{5}$ Department of Dermatology, University Hospital Münster, Münster, Germany
${ }^{6}$ Department of Dermatology, Saarland University Medical Center, Homburg/Saar,
Germany
${ }^{7}$ Department of Dermatology, University Hospital Würzburg, Würzburg, Germany
${ }^{8}$ Department of Dermatology, Helios Klinikum Erfurt, Erfurt, Germany
${ }^{9}$ Department of Dermatology, University Hospital Kiel, Kiel, Germany
${ }^{10}$ Department of Dermatology, University Hospital Tübingen, Tübingen, Germany
${ }^{11}$ Department of Dermatology, Ludwig-Maximilians University Munich, Munich,
Germany
${ }^{12}$ Department of Dermatology, University Medical Center Mainz, Mainz, Germany

\section{Author affiliations}

Essen, Essen, Germany

Epidemiology, University of Duisburg-Essen, Essen, Germany

${ }^{3}$ Skin Cancer Center Hannover, Department of Dermatology, Hannover Medical

School, Hannover, Germany

${ }^{4}$ Department of Dermatology, University Hospital Erlangen, Erlangen, Germany ${ }^{6}$ Department of Dermatology, Saarland University Medical Center, Homburg/Saa Germany

${ }^{8}$ Department of Dermatology, Helios Klinikum Erfurt, Erfurt, Germany

${ }^{9}$ Department of Dermatology, University Hospital Kiel, Kiel, Germany

${ }^{10}$ Department of Dermatology, University Hospital Tübingen, Tübingen, Germany

Germany

${ }^{12}$ Department of Dermatology, University Medical Center Mainz, Mainz, Germany 
${ }^{13}$ Skin Cancer Unit, German Cancer Research Center (DKFZ) and Department of Dermatology, Venereology and Allergology, niversity Medical Center Mannheim, Ruprecht-Karl University of Heidelberg, Mannheim, Germany

${ }^{14}$ Department of Dermatology, University Hospital Lübeck, Lübeck, Germany

${ }^{15}$ Department of Dermatology, Wald-Klinikum Gera, Gera, Germany

${ }^{16}$ Department of Dermatology, Skin Cancer Center at CIO Koeln/Bonn, University

Hospital Cologne, Cologne, Germany

${ }^{17}$ Department of Dermatology, Helios St. Elisabeth Hospital Oberhausen, University

of Witten-Herdecke, Oberhausen, Germany

${ }^{18}$ Department of Dermatology, Klinikum Quedlinburg, Quedlinburg, Germany

${ }^{19}$ Department of Dermatology, Elbe-Klinikum Buxtehude, Buxtehude, Germany

${ }^{20}$ Department of Dermatology, Klinikum Ludwigshafen, Ludwigshafen, Germany

${ }^{21}$ Translational Skin Cancer Research, Deutsches Konsortium für Translationale Krebsforschung (DKTK), Deutsches Konsortium für Translationale Krebsforschung (DKTK), Essen, Germany

${ }^{22}$ Department of Neurology, University Hospital Essen, University of Duisburg-Essen, Essen, Germany

Acknowledgements We wish to thank all patients participating in this study, as well as their families and caregivers.

Contributors Study conception and design, writing of manuscript: SK, AS, SU. Data acquisition: SK, LZ, RG, LH, CW, CP, AG, RH, KCK, BW, CB, CL, JU, PT, MK, MS, AK, JU, PM, ED, EL, JCB, MW, EC, DS, SU. Data analysis and interpretation: SK, AS, HL, JG, SU. Review and approval of manuscript: All authors.

Funding This study was performed within the network of study centers of the DeCOG.

Competing interests SK has received travel support from Bristol-Myers Squibb and Amgen. AS received speaker honoraria from Merck Serono. LZ has served as consultant and/or has received honoraria from Roche, Bristol-Myers Squibb, Merck Sharp and Dohme, Novartis, Pierre Fabre, and Sanofi and has received trave support from Bristol-Myers Squibb, Merck Sharp and Dohme, Amgen, Pierre Fabre and Novartis. RG received honoraria for lectures and advisory boards, research support and meeting support from Almirall Hermal, Amgen, Astra Zeneca, BristolMyers Squibb, Leo, Merck Serono, Merck Sharp and Dohme, Pierre Fabre, Roche, Sanofi Genzyme, Regeneron, Sun Pharma, Takeda, Pfizer, Novartis, Johnson \& Johnson, 4SC, and Incyte. LH has received grants from Novartis and has received personal fees from Amgen, Bristol-Myers Squibb, Merck Sharp and Dohme, Roche, Curevac, Pierre Fabre, Novartis and Sanofi. CW has served as consultant and/or has received honoraria from Amgen, Bristol-Myers Squibb, Curevac, Merck Sharp and Dohme, Novartis, Pierre Fabre, Roche, Sanofi, and Takeda, and has received travel support from Leo and Teva. CP received speaker or consultant honoraria and travel support from Novartis, Bristol-Myers Squibb, Roche, Merck Serono, Merck Sharp and Dohme, Celgene, AbbVie and LEO. AG reports speakers honoraria from BristolMyers Squibb, Merck Sharp and Dohme, and Roche; advisory board honoraria from Bristol-Myers Squibb, Novartis, Merck Sharp and Dohme, Pierre Fabre, Pfizer, Roche and Sanofi Genzyme; and travel support from Bristol-Myers Squibb, Merck Sharp and Dohme, Novartis, and Roche. RH declares speakers and advisory board honoraria from Bristol-Myers Squibb, Merck Sharp and Dohme, Novartis, and Roche. KCK has served as consultant and/or has received honoraria from Amgen, Roche, Bristol-Myers Squibb, Merck Sharp and Dohme, Pierre Fabre, and Novartis and has received travel support from Amgen, Merck Sharp and Dohme, BristolMyers Squibb, Amgen, Pierre Fabre, Medac, and Novartis. BW reports grants and personal fees from Bristol-Myers Squibb, Philogen and Merck Sharp and Dohme, and personal fees from Roche, Novartis, and Curevac. CB reports grants, personal fees, and non-financial support from Amgen, Bristol-Myers Squibb, Merck Sharp and Dohme, Merck Serono, Novartis, Roche, and 4SC, personal fees and nonfinancial support from Pierre Fabre and Sanofi Aventis as well as grants from Array Pharma and Regeneron. CL declares speakers and advisory board honoraria and travel support from Bristol-Myers Squibb, Merck Sharp and Dohme, Merck Serono, Novartis, Roche, Amgen, Pierre Fabre, and Sun Pharma. JU is on the advisory board or has received honoraria and travel support from Amgen, Bristol-Myers Squibb, GlaxoSmithKline, Leo, Merck Sharp and Dohme, Novartis, Pierre Fabre, and Roche. PT has received speakers honoraria from Bristol-Myers Squibb, Novartis, Pierre Fabre and Roche, consultants honoraria from Bristol-Myers Squibb, Merck Serono, Novartis, Pierre Fabre and Roche, and travel support from Bristol-Myers Squibb and Pierre-Fabre. MK has received grants from Bristol-Myers Squibb, Merck Sharp and Dohme, Leo, Novartis and Roche. MS received honoraria for lectures from Merck Sharp and Dohme, Bristol-Myers Squibb, Roche, and Pierre Fabre, and served on advisory boards for Novartis, Roche, Merck Sharp and Dohme, and Pierre-Fabre. AK declares advisory board and speakers honoraria from Merck Sharp and Dohme,
Sanofi Pasteur, and AbbVie. JU declares research support from Novartis, speakers and advisory board honoraria from Bristol-Myers Squibb, Merck Sharp and Dohme, Novartis, Roche and Sanofi, and travel support from Bristol Myers Squibb and Medac. PM declares research support from Bristol-Myers Squibb and Merck Sharp and Dohme, speakers and advisory board honoraria from Amgen, Bristol-Myers Squibb, Merck Sharp and Dohme, Merck Serono, Sanofi, Novartis, Roche, and Pierre Fabre, and travel support from Bristol-Myers Squibb, Merck Sharp and Dohme, Pierre Fabre and Novartis. EL has served as consultant or/and has received honoraria from Amgen, Actelion, Roche, Bristol-Myers Squibb, Merck Sharp and Dohme, Novartis, Janssen, Medac, and travel support from Amgen, Merck Sharp and Dohme, Bristol-Myers Squibb, Amgen, Pierre Fabre, and Novartis. JCB has received speakers honoraria from Amgen, MerckSerono, and Pfizer, advisory board honoraria from 4SC, Amgen, CureVac, eTheRNA, Lytix, MerckSerono, Novartis, Pfizer, Rigontec, and Sanofi as well as research funding from Alcedis, Bristol-Myers Squibb, Boehringer Ingelheim, IQVIA, and Merck Serono; he also received travel support from 4SC and Incyte. MW declares speakers and advisory board honoraria from Merck, Bristol-Myers Squibb, Roche, Novartis, Sanofi, Pierre Fabre, Beiersdorf, Sun Pharma, and Takeda. EC received travel support from Bristol-Myers Squibb, Merck Sharp and Dohme, and Novartis. DS declares advisory board and speakers honoraria from Roche, Novartis, Bristol-Myers Squibb, Merck, Amgen, Boehringer Ingelheim and Leo, as well as grant and travel support from Roche, Novartis, Bristol-Myers Squibb, Merck, Amgen, Boehringer Ingelheim and Leo. SU declares research support from Bristol-Myers Squibb and Merck Serono, speakers and advisory board honoraria from Bristol-Myers Squibb, Merck Sharp and Dohme, Merck Serono, Novartis, and Roche, and travel support from Bristol-Myers Squibb, Merck Sharp and Dohme. All remaining authors declared no conflicts of interest.

Patient consent for publication Not required.

Ethics approval The study was approved by the ethics committee of the University Duisburg-Essen (17-7743-B0).

Provenance and peer review Not commissioned; externally peer reviewed

Data availability statement All patients are annotated for tumor characteristics, course of disease, as well as therapy characteristics and outcome. These detailed data are available from the corresponding author on reasonable request.

Open access This is an open access article distributed in accordance with the Creative Commons Attribution Non Commercial (CC BY-NC 4.0) license, which permits others to distribute, remix, adapt, build upon this work non-commercially, and license their derivative works on different terms, provided the original work is properly cited, appropriate credit is given, any changes made indicated, and the use is non-commercial. See http://creativecommons.org/licenses/by-nc/4.0/.

\section{ORCID iDs}

Jürgen C Becker http://orcid.org/0000-0001-9183-653X

Michael Weichenthal http://orcid.org/0000-0002-9060-4961

Selma Ugurel http://orcid.org/0000-0002-9384-6704

\section{REFERENCES}

1 Schadendorf D, van Akkooi ACJ, Berking C, et al. Melanoma. Lancet 2018;392:971-84.

2 Ugurel S, Röhmel J, Ascierto PA, et al. Survival of patients with advanced metastatic melanoma: the impact of novel therapiesupdate 2017. Eur J Cancer 2017;83:247-57.

3 Schadendorf D, Hodi FS, Robert C, et al. Pooled analysis of long-term survival data from phase II and phase III trials of ipilimumab in unresectable or metastatic melanoma. J Clin Oncol 2015;33:1889-94.

4 Hodi FS, Chiarion-Sileni V, Gonzalez R, et al. Nivolumab plus ipilimumab or nivolumab alone versus ipilimumab alone in advanced melanoma (CheckMate 067): 4-year outcomes of a multicentre, randomised, phase 3 trial. Lancet Oncol 2018;19:1480-92.

5 Hamid O, Robert C, Daud A, et al. Five-Year survival outcomes for patients with advanced melanoma treated with pembrolizumab in KEYNOTE-001. Ann Oncol 2019;30:582-8.

6 Jacquelot N, Roberti MP, Enot DP, et al. Predictors of responses to immune checkpoint blockade in advanced melanoma. Nat Commun 2017;8:592.

7 Axelrod ML, Johnson DB, Balko JM. Emerging biomarkers for cancer immunotherapy in melanoma. Semin Cancer Biol 2018;52:207-15.

8 Shaverdian N, Lisberg AE, Bornazyan K, et al. Previous radiotherapy and the clinical activity and toxicity of pembrolizumab in the treatment of non-small-cell lung cancer: a secondary analysis of the KEYNOTE-001 phase 1 trial. Lancet Oncol 2017;18:895-903. 
9 Chicas-Sett R, Morales-Orue I, Castilla-Martinez J, et al. Stereotactic ablative radiotherapy combined with immune checkpoint inhibitors reboots the immune response assisted by immunotherapy in metastatic lung cancer: a systematic review. Int $J$ Mol Sci 2019;20. doi:10.3390/ijms20092173. [Epub ahead of print: 02 May 2019].

10 Sridharan V, Margalit DN, Lynch SA, et al. Definitive chemoradiation alters the immunologic landscape and immune checkpoints in head and neck cancer. Br J Cancer 2016;115:252-60.

11 Golden EB, Chhabra A, Chachoua A, et al. Local radiotherapy and granulocyte-macrophage colony-stimulating factor to generate abscopal responses in patients with metastatic solid tumours: a proof-of-principle trial. Lancet Oncol 2015;16:795-803.

12 Sharma A, Bode B, Studer G, et al. Radiotherapy of human sarcoma promotes an intratumoral immune effector signature. Clin Cancer Res 2013;19:4843-53.

13 Reits EA, Hodge JW, Herberts CA, et al. Radiation modulates the peptide repertoire, enhances MHC class I expression, and induces successful antitumor immunotherapy. J Exp Med 2006;203:1259-71.

14 Jiang W, Chan CK, Weissman IL, et al. Immune priming of the tumor microenvironment by radiation. Trends Cancer 2016;2:638-45.

15 Weichselbaum RR, Liang H, Deng L, et al. Radiotherapy and immunotherapy: a beneficial liaison? Nat Rev Clin Oncol 2017;14:365-79.

16 Buchwald ZS, Wynne J, Nasti TH, et al. Radiation, immune checkpoint blockade and the abscopal effect: a critical review on timing, dose and fractionation. Front Oncol 2018:8:612.

17 Shabason JE, Minn AJ. Radiation and immune checkpoint blockade: from bench to clinic. Semin Radiat Oncol 2017;27:289-98.

18 Chicas-Sett R, Morales-Orue I, Rodriguez-Abreu D, et al. Combining radiotherapy and ipilimumab induces clinically relevant radiationinduced abscopal effects in metastatic melanoma patients: a systematic review. Clin Transl Radiat Oncol 2018;9:5-11.

19 Gershenwald JE, Scolyer RA, Hess KR, et al. Melanoma staging: evidence-based changes in the American joint Committee on cancer eighth edition cancer staging manual. CA Cancer J Clin 2017;67:472-92.

20 Therasse P, Arbuck SG, Eisenhauer EA, et al. New guidelines to evaluate the response to treatment in solid tumors. European organization for research and treatment of cancer, National cancer Institute of the United States, National cancer Institute of Canada. J Natl Cancer Inst 2000;92:205-16.

21 Cole SR, Hernán MA. Adjusted survival curves with inverse probability weights. Comput Methods Programs Biomed 2004;75:45-9.

22 Pearl J. An introduction to causal inference. Int J Biostat 2010;6:Article 7. Article 7.

23 Sevenich L. Turning "Cold" Into "Hot" Tumors-Opportunities and Challenges for Radio-Immunotherapy Against Primary and Metastatic Brain Cancers. Front Oncol 2019;9:163.

24 Postow MA, Callahan MK, Barker CA, et al. Immunologic correlates of the abscopal effect in a patient with melanoma. $N$ Engl $J$ Med 2012:366:925-31.

25 Grimaldi AM, Simeone E, Giannarelli D, et al. Abscopal effects of radiotherapy on advanced melanoma patients who progressed after ipilimumab immunotherapy. Oncoimmunology 2014;3:e28780.

26 Twyman-Saint Victor C, Rech AJ, Maity A, et al. Radiation and dual checkpoint blockade activate non-redundant immune mechanisms in cancer. Nature 2015;520:373-7.

27 Tawbi HA, Forsyth PA, Algazi A, et al. Combined nivolumab and ipilimumab in melanoma metastatic to the brain. N Engl J Med 2018;379:722-30.

28 Long GV, Atkinson V, Lo S, et al. Combination nivolumab and ipilimumab or nivolumab alone in melanoma brain metastases: a multicentre randomised phase 2 study. Lancet Oncol 2018:19:672-81.

29 Khozin S, Blumenthal GM, Pazdur R. Real-World data for clinical evidence generation in oncology. J Natl Cancer Inst 2017;109. doi:10.1093/jnci/djx187. [Epub ahead of print: 01 Nov 2017].

30 Knisely JPS, Yu JB, Flanigan J, et al. Radiosurgery for melanoma brain metastases in the ipilimumab era and the possibility of longer survival. J Neurosurg 2012;117:227-33.

31 Barker CA, Postow MA, Khan SA, et al. Concurrent radiotherapy and ipilimumab immunotherapy for patients with melanoma. Cancer Immunol Res 2013;1:92-8.
32 Mathew M, Tam M, Ott PA, et al. Ipilimumab in melanoma with limited brain metastases treated with stereotactic radiosurgery. Melanoma Res 2013;23:191-5.

33 Silk AW, Bassetti MF, West BT, et al. Ipilimumab and radiation therapy for melanoma brain metastases. Cancer Med 2013;2:899-906.

34 Chandra RA, Wilhite TJ, Balboni TA, et al. A systematic evaluation of abscopal responses following radiotherapy in patients with metastatic melanoma treated with ipilimumab. Oncoimmunology 2015;4:e1046028.

35 Kiess AP, Wolchok JD, Barker CA, et al. Stereotactic radiosurgery for melanoma brain metastases in patients receiving ipilimumab: safety profile and efficacy of combined treatment. Int J Radiat Oncol Biol Phys 2015;92:368-75.

36 Tazi K, Hathaway A, Chiuzan C, et al. Survival of melanoma patients with brain metastases treated with ipilimumab and stereotactic radiosurgery. Cancer Med 2015;4:1-6.

37 Twyman-Saint Victor C, Rech AJ, Maity A, et al. Radiation and dual checkpoint blockade activate non-redundant immune mechanisms in cancer. Nature 2015;520:373-7.

38 Hiniker SM, Reddy SA, Maecker HT, et al. A Prospective Clinical Trial Combining Radiation Therapy With Systemic Immunotherapy in Metastatic Melanoma. Int J Radiat Oncol Biol Phys 2016;96:578-88.

39 Qian JM, Yu JB, Kluger HM, et al. Timing and type of immune checkpoint therapy affect the early radiographic response of melanoma brain metastases to stereotactic radiosurgery. Cancer 2016;122:3051-8.

40 Qin R, Olson A, Singh B, et al. Safety and efficacy of radiation therapy in advanced melanoma patients treated with ipilimumab. Int J Radiat Oncol Biol Phys 2016;96:72-7.

41 Theurich S, Rothschild SI, Hoffmann M, et al. Local tumor treatment in combination with systemic ipilimumab immunotherapy prolongs overall survival in patients with advanced malignant melanoma. Cancer Immunol Res 2016;4:744-54.

42 Koller KM, Mackley HB, Liu J, et al. Improved survival and complete response rates in patients with advanced melanoma treated with concurrent ipilimumab and radiotherapy versus ipilimumab alone. Cancer Biol Ther 2017:18:36-42.

43 Patel KR, Shoukat S, Oliver DE, et al. Ipilimumab and stereotactic radiosurgery versus stereotactic radiosurgery alone for newly diagnosed melanoma brain metastases. Am J Clin Oncol 2017;40:444-50.

44 Minniti G, Anzellini D, Reverberi C, et al. Stereotactic radiosurgery combined with nivolumab or ipilimumab for patients with melanoma brain metastases: evaluation of brain control and toxicity. $J$ Immunother Cancer 2019;7:102.

45 Ahmed KA, Stallworth DG, Kim Y, et al. Clinical outcomes of melanoma brain metastases treated with stereotactic radiation and anti-PD-1 therapy. Ann Oncol 2016:27:434-41.

46 Liniker E, Menzies AM, Kong BY, et al. Activity and safety of radiotherapy with anti-PD-1 drug therapy in patients with metastatic melanoma. Oncoimmunology 2016;5:e1214788.

47 Aboudaram A, Modesto A, Chaltiel L, et al. Concurrent radiotherapy for patients with metastatic melanoma and receiving antiprogrammed-death 1 therapy: a safe and effective combination. Melanoma Res 2017;27:485-91.

48 Anderson ES, Postow MA, Wolchok JD, et al. Melanoma brain metastases treated with stereotactic radiosurgery and concurrent pembrolizumab display marked regression; efficacy and safety of combined treatment. J Immunother Cancer 2017;5:76.

49 Pike LRG, Bang A, Ott P, et al. Radiation and PD-1 inhibition: favorable outcomes after brain-directed radiation. Radiother Oncol 2017;124:98-103.

50 Maity A, Mick R, Huang AC, et al. A phase I trial of pembrolizumab with hypofractionated radiotherapy in patients with metastatic solid tumours. Br J Cancer 2018;119:1200-7.

51 Nardin C, Mateus C, Texier M, et al. Tolerance and outcomes of stereotactic radiosurgery combined with anti-programmed cell death-1 (pembrolizumab) for melanoma brain metastases. Melanoma Res 2018;28:111-9.

52 Roger A, Finet A, Boru B, et al. Efficacy of combined hypofractionated radiotherapy and anti-PD-1 monotherapy in difficult-to-treat advanced melanoma patients. Oncoimmunology 2018;7:e1442166.

53 Trommer-Nestler M, Marnitz S, Kocher M, et al. Robotic stereotactic radiosurgery in melanoma patients with brain metastases under simultaneous anti-PD-1 treatment. Int J Mol Sci 2018;19:11. 